\title{
A recently transferred cluster of bacterial genes in Trichomonas vaginalis - lateral gene transfer and the fate of acquired genes
}

\author{
Åke Strese ${ }^{1}$, Anders Backlund ${ }^{1}$ and Cecilia Alsmark ${ }^{1,2^{*}}$
}

\begin{abstract}
Background: Lateral Gene Transfer (LGT) has recently gained recognition as an important contributor to some eukaryote proteomes, but the mechanisms of acquisition and fixation in eukaryotic genomes are still uncertain. A previously defined norm for LGTs in microbial eukaryotes states that the majority are genes involved in metabolism, the LGTs are typically localized one by one, surrounded by vertically inherited genes on the chromosome, and phylogenetics shows that a broad collection of bacterial lineages have contributed to the transferome.

Results: A unique $34 \mathrm{kbp}$ long fragment with 27 clustered genes (TVLF) of prokaryote origin was identified in the sequenced genome of the protozoan parasite Trichomonas vaginalis. Using a PCR based approach we confirmed the presence of the orthologous fragment in four additional T. vaginalis strains. Detailed sequence analyses unambiguously suggest that TVLF is the result of one single, recent LGT event. The proposed donor is a close relative to the firmicute bacterium Peptoniphilus harei. High nucleotide sequence similarity between $T$. vaginalis strains, as well as to $P$. harei, and the absence of homologs in other Trichomonas species, suggests that the transfer event took place after the radiation of the genus Trichomonas. Some genes have undergone pseudogenization and degradation, indicating that they may not be retained in the future. Functional annotations reveal that genes involved in informational processes are particularly prone to degradation.
\end{abstract}

Conclusions: We conclude that, although the majority of eukaryote LGTs are single gene occurrences, they may be acquired in clusters of several genes that are subsequently cleansed of evolutionarily less advantageous genes.

Keywords: Lateral gene transfer (LGT), Trichomonas, Peptoniphilus, Phylogeny

\section{Background}

The protozoan parasite Trichomonas vaginalis is a human pathogen that causes the most common, non-viral, sexually transmitted disease in the world, infecting 248 million people yearly according to WHO estimates [1]. Men are often asymptomatic carriers of the parasite, while symptoms in women range from malodorous vaginal discharge, inflammation and swelling of the urogenital tract to increased risk for cervical cancer, adverse pregnancy outcomes and an increased susceptibility to HIV-1 infection [2-4]. Treatment today is limited to two nitroimidazole derivatives, tinidazole and metronidazole, although failure

\footnotetext{
* Correspondence: cecilia.alsmark@fkog.uu.se

'Division of Pharmacognosy, Department of Medicinal Chemistry, Uppsala University, Uppsala, Sweden

${ }^{2}$ Department of Virology, Immunobiology and Parasitology, National Veterinary Institute, Uppsala, Sweden
}

of treatment due to resistance has been reported [5]. A draft genome sequence of $T$. vaginalis G3 was accomplished in 2007 [6], revealing an unusually large genome of more than $160 \mathrm{Mbp}$, encoding up to 60,000 genes in addition to numerous and diverse repeated regions.

LGT is the acquisition and fixation in the recipient genome of genetic material from a foreign donor organism without sexual transfer. It offers a rapid retrieval of new capabilities such as the ability to utilize new metabolites [7], degradation of chemicals such as pesticides [8] or the deployment of drug resistance genes [9]. The bacterial routes for uptake of foreign DNA are well described by features such as transformation, conjugation and transduction, or by the activities of "gene transfer agents" such as transposable elements. The mechanisms for eukaryotic gene acquisition are less well described [10], although one of the favored hypothesis suggests that the transfer is 
mediated via phagocytosis [11]. Recent finds, however, assume that e.g. transposable elements may facilitate uptake of prokaryote genes also in unicellular eukaryotes [12].

Laterally transferred genes have previously been identified with a phylogenomic approach in T. vaginalis $[13,14]$, estimating that $0.25 \%$ of the genes in the genome were acquired by LGT from prokaryote or non-related eukaryote sources. These analyses in T. vaginalis and other protozoa also show that there are common features that hold true for the majority of genes acquired via LGT in unicellular eukaryotes [15]. One such feature is that LGTs are typically operational, rather than informational genes, a notion that has been predicted previously [16]. Another feature is that rather than one single bacterial donor lineage, as is the case for mitochondrial genes originating from an $\alpha$-proteobacterial source, a broad range of prokaryotic donor organisms was identified. Furthermore, the LGTs in protozoa are typically not physically linked together on the recipient chromosome, but scattered between vertically inherited genes [17]. The haploid genome [14] of $T$. vaginalis, in combination with the assumption of an asexual reproduction [18], leaves this parasite with ample possibilities for fixation of acquired genes, since all genetic material within the nucleus is passed to the new generation following division [19]. Also, being unicellular, all new genetic material that is successfully incorporated in the genome of $T$. vaginalis will be passed on to all offspring following reproduction. In higher eukaryotes with multiple cells, however, a change in the genome must be fixated in a gamete in order to be passed on to its offspring.

In stark contrast to the observations of the norm for LGT in eukaryotes, the genome of $T$. vaginalis harbors a region of $34 \mathrm{kbp}$ located on contig DS113827, at positions $18,333-52,058$, that exhibits highly unusual characteristics. Our bioinformatic assessment based on similarity searches and phylogenetic analyses indicates that a recent transfer event may be the cause of this anomaly. This region, designated as the Trichomonas vaginalis Lateral gene transfer Fragment (TvLF), has been the target for in-depth analyses applying bioinformatic and molecular biology tools, with the purpose and aim to shed light on the comparably unknown features involved in the acquisition of LGT in eukaryotes.

\section{Results}

Confirming authenticity of TVLF as a part of the $T$. vaginalis genome

Compared to previously detected LGTs in parasites $[13,20]$, the genes on TvLF have unusually high DNA sequence similarity to the putative donor Peptoniphilus harei (a firmicute bacterium), with no less than 79-98\% identity at the amino acid level. An overview of the TvLF in comparison to the corresponding fragment in $P$. harei is visualized in the gene-map (Figure 1).

Such high identity levels are often considered to result from contaminants in the DNA source [21]. In this particular case, however, we present firm evidence providing an alternative explanation due to LGT. First, the genes of TvLF are not similar to known genes of Mycoplasma, a bacterium known to be frequently associated with, infect and multiply within $T$. vaginalis cultures [22-24]. Second, the $T$. vaginalis $\mathrm{G} 3$ strain used to sequence the complete genome was grown as an axenic culture [14]. Third, in addition to the putative LGTs the scaffold DS113827 containing the TvLF also encodes three consecutive genes, which are so far unique to T. vaginalis, TVAG_243540, TVAG_243550 and TVAG_243560. All three of these genes are repeated and dispersed also in other loci throughout the T. vaginalis G3 genome [14,25], as summarized in Additional file 1: Table S1.

To further establish that the genes on TvLF are genuinely part of the $T$. vaginalis genome, the physical connection between the $T$. vaginalis-specific repeated genes and the bacteria-like genes were re-confirmed by PCR and sequencing. During this process the size and nucleotide sequence of all annotated sequence gaps in the TvLF-region was determined (Additional file 1: Table S2). In addition, we have successfully amplified, using PCR, the proposed LGTs on TvLF in four additional strains of $T$. vaginalis (Table 1). Furthermore, the TvLF is present in the T. vaginalis G3 strain sequenced by the gene index project at TIGR. We consider the likelihood of the same contaminant affecting isolates maintained in three separate laboratories to be very small.

However, to further rule out contamination, the presence of three randomly selected TvLF genes (TVAG_243650, 243760 and 243820) was confirmed in two additional strains ( $T$. vaginalis $\mathrm{T} 1$ and $T$. vaginalis $\mathrm{P} 9$ ) that were not used for further sequence analysis. These seven isolates were maintained by three independent laboratories. Furthermore, PCR primers retrieved from the literature were used in an ultimately futile attempt to amplify regions of the $16 \mathrm{~s}$-rDNA, to detect any bacterial contaminant in our sources of $T$. vaginalis DNA (Additional file 1: Table S3). Repeated attempts failed to amplify any products with primer pair $16 \mathrm{~s}: 1$, but pair $16 \mathrm{~s}: 2$ amplified the $18 \mathrm{~S}$ ribosomal RNA gene of $T$. vaginalis (accession AY338475.1). No amplicons similar to any bacterial $16 \mathrm{~S}$ rRNA genes were produced. In addition, fluorescence in situ hybridizations of probes within the TvLF to T. vaginalis nuclei confirm the presence of TvLF on one single locus in the T. vaginalis genome (Alsmark, unpublished data).

The TvLF appears to be specific to $T$. vaginalis, as none of the TvLF-genes could be identified by PCR in either Trichomonas gallinae or Trichomonas tenax, using primer sequences conserved in both $T$. vaginalis and 


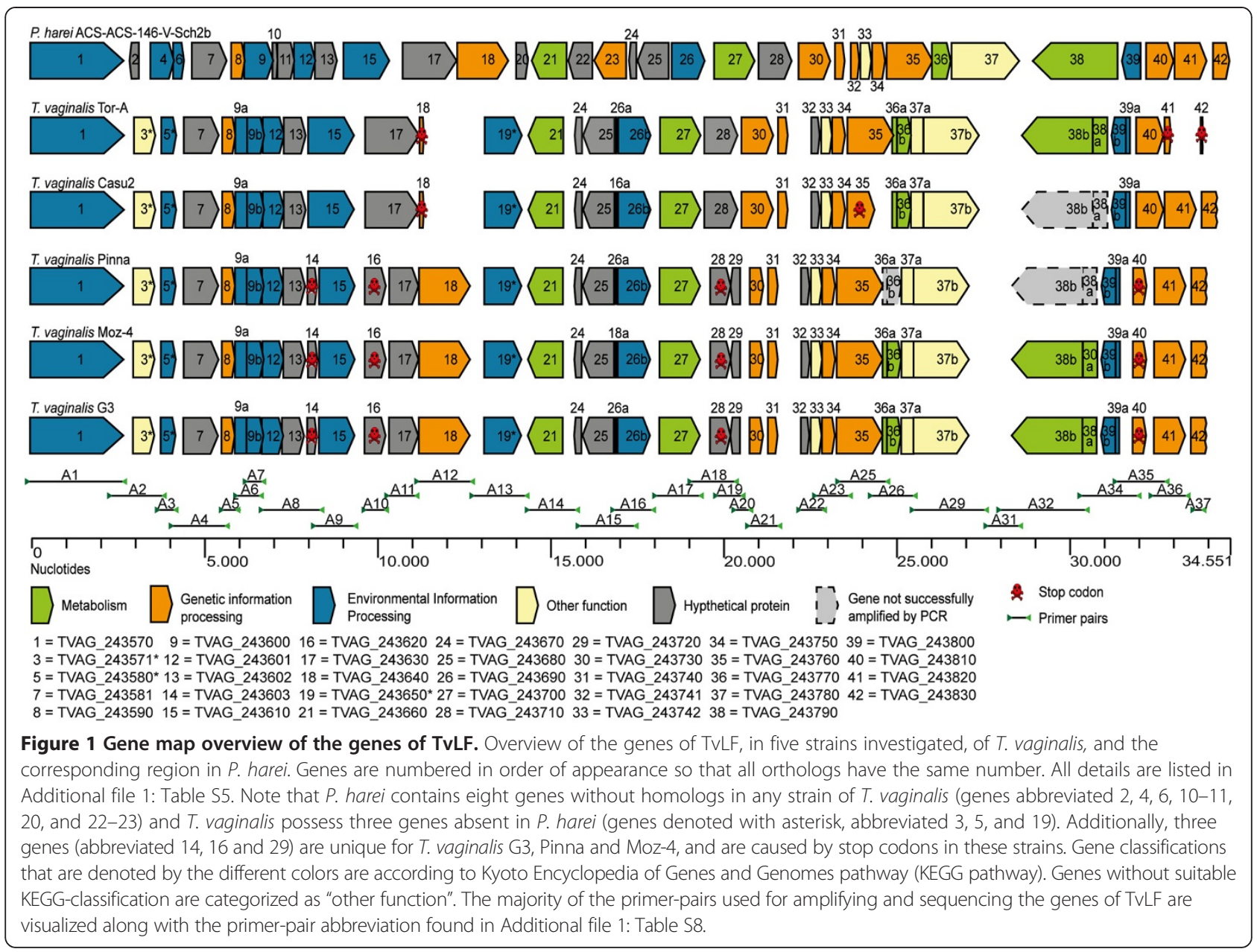

$P$. harei. In previous studies $T$. gallinae and $T$. tenax have been verified to be the two most closely related species to $T$. vaginalis within the class of Trichomonadea [26]. This indicates that the transfer has occurred after the divergence of $T$. vaginalis from the remainder of the genus. A recent acquisition would be in agreement with the

Table 1 Identification of $T$. vaginalis strains used in this study

\begin{tabular}{|c|c|c|}
\hline Strain & Isolated & Location \\
\hline$\overline{G 3}$ (PRA98) $^{1}$ & 1973 & Beckham, United Kingdom \\
\hline Casu2 $(\text { SS-22) })^{1}$ & 2008 & Sardinia, Italy \\
\hline Moz-4 (MPM4) ${ }^{1}$ & 1997 & Mozambique \\
\hline Pinna $(\text { SS-28) })^{1}$ & 1998 & Sardinia, Italy \\
\hline Tor-A $\left(\right.$ TO-01) ${ }^{1}$ & 2010 & Turin, Italy \\
\hline $\mathrm{T} 1^{2}$ & 1993 & Taipei, Taiwan \\
\hline$P 9^{2}$ & - & Prague, Czech Republic \\
\hline
\end{tabular}

${ }^{1}$ Strains used in this study to investigate TvLF.

${ }^{2}$ Strains tested positive for the presence of three randomly selected TVLF genes. unusually high nucleotide sequence similarity to orthologs of the putative bacterial donor (Table 2).

\section{The genomic architecture of TvLF}

The genes on TvLF encompass a stretch of 27 consecutive genes of bacterial origin, TVAG_243570-TVAG_243830, spanning more than $34 \mathrm{kbp}$ of the $52 \mathrm{kbp}$ long contig DS113827 in the T. vaginalis G3 genome (Figure 1, Table 2 and Additional file 1: Table S4 and Additional file 1: Table S5). Although absent from the sequenced eukaryote gene-pool, an homologous region was detected in the firmicute bacterium Peptoniphilus harei (contig 0004, positions 22397-56995, HMPREF9286_0330-HMPREF9 286_0294, reverse direction). The TvLF stands in contrast to other LGTs detected in parasite genomes that typically are singletons embedded among vertically inherited genes $[17,27]$.

A comprehensive comparative sequence analysis of the TvLF in T. vaginalis G3 and the putative bacterial donor reveals an unusually high degree of nucleotide sequence similarity (79-98\%), compared to that of typical prokaryote- 
Table 2 Genes of TvLF

\begin{tabular}{|c|c|c|c|c|c|}
\hline $\begin{array}{l}\text { TvLF gene } \\
\text { abbreviation }\end{array}$ & Annotated function & $\begin{array}{l}\text { Top blastx } \\
\text { hit }^{1}\end{array}$ & $\begin{array}{l}\text { Query } \\
\text { coverage }\end{array}$ & $\begin{array}{l}\text { Max } \\
\text { identity }\end{array}$ & $\begin{array}{l}\text { GenBank } \\
\text { accession no. }\end{array}$ \\
\hline TVAG_243570 & Cation transporting E1-E2 ATPase & P. harei & $99 \%$ & $96 \%$ & ZP_07822791.1 \\
\hline TVAG_243580 & Transposase IS116/IS110/IS902 family protein & S. pyogenes & $99 \%$ & $94 \%$ & CAQ56293.1 \\
\hline TVAG_243590 & Transcriptional regulator, gntR family protein & P. harei & $99 \%$ & $92 \%$ & ZP_07822817.1 \\
\hline TVAG_243600 & $\mathrm{ABC}$ transporter & P. harei & $99 \%$ & $98 \%$ & ZP_07822813.1 \\
\hline TVAG_243610 & Xanthine/uracil purine permease family protein & P. harei & $99 \%$ & $98 \%$ & ZP_07822771.1 \\
\hline TVAG_243620 & Conserved hypothetical protein & P. harei & $99 \%$ & $90 \%$ & ZP_07822780.1 \\
\hline TVAG_243630 & S-layer homology domain containing protein & P. harei & $99 \%$ & $94 \%$ & ZP_07822780.1 \\
\hline TVAG_243640 & Glutamyl-tRNA synthetase & P. harei & $99 \%$ & $96 \%$ & ZP_07822745.1 \\
\hline TVAG_243650 & Transposase family protein & P. sp. & $49 \%$ & $92 \%$ & ZP_07094114.1 \\
\hline TVAG_243660 & Chitooligosaccharide deacetylase & P. harei & $99 \%$ & $92 \%$ & ZP_07822823.1 \\
\hline TVAG_243670 & Conserved hypothetical protein & P. harei & $98 \%$ & $90 \%$ & ZP_07822757.1 \\
\hline TVAG_243680 & Conserved hypothetical protein & P. harei & $99 \%$ & $91 \%$ & ZP_07822760.1 \\
\hline TVAG_243690 & Auxin Efflux Carrier family protein & P. harei & $99 \%$ & $91 \%$ & ZP_07822772.1 \\
\hline TVAG_243700 & S-adenosylmethionine synthetase & P. harei & $99 \%$ & $97 \%$ & ZP_07822781.1 \\
\hline TVAG_243710 & Hypothetical protein & P. harei & $85 \%$ & $89 \%$ & ZP_07822767.1 \\
\hline TVAG_243720 & Conserved hypothetical protein & P. harei & $94 \%$ & $99 \%$ & ZP_07822767.1 \\
\hline TVAG_243730 & Conserved hypothetical protein & P. harei & $89 \%$ & $92 \%$ & ZP_07822775.1 \\
\hline TVAG_243740 & DNA-binding protein $\mathrm{HU}$ & P. harei & $62 \%$ & $99 \%$ & ZP_07822787.1 \\
\hline TVAG_243750 & $\begin{array}{l}\text { S1 RNA binding domain containing protein, polyribonucleotide } \\
\text { nucleotidyltransferase }\end{array}$ & P. harei & $99 \%$ & $95 \%$ & ZP_07822810.1 \\
\hline TVAG_243760 & PP-loop family protein, cell cycle protein & P. harei & $99 \%$ & $90 \%$ & ZP_07822759.1 \\
\hline TVAG_243770 & Hypoxanthine-guanine phosphoribosyltransferase & P. harei & $99 \%$ & $96 \%$ & ZP_07822797.1 \\
\hline TVAG_243780 & Clan MA, family M41, FtsH endopeptidase-like metallopeptidase & P. harei & $96 \%$ & $96 \%$ & ZP_07822796.1 \\
\hline TVAG_243790 & Penicillin binding protein transpeptidase, & P. harei & $90 \%$ & $97 \%$ & ZP_07822802.1 \\
\hline TVAG_243800 & BioY family protein & P. harei & $82 \%$ & $93 \%$ & ZP_07822776.1 \\
\hline TVAG_243810 & $\begin{array}{l}\text { Conserved hypothetical protein (positive regulation of transcription, } \\
\text { DNA-dependent) }\end{array}$ & P. harei & $95 \%$ & $99 \%$ & ZP_07822814.1 \\
\hline TVAG_243820 & tRNA-dihydrouridine synthase & P. harei & $99 \%$ & $96 \%$ & ZP_07822761.1 \\
\hline TVAG_243830 & Transcription elongation factor greA & P. harei & $95 \%$ & $97 \%$ & ZP_07822812.1 \\
\hline
\end{tabular}

${ }^{1} P$. harei $=$ Peptoniphilus harei. S. pyogenes $=$ Streptococcus pyogenes. $P$. sp. $=$ Peptoniphilus species oral taxon 836.

to-protozoa LGTs detected previously $(27-83 \%)$ [14,17,28]. Comparing the gene order of TvLF with the corresponding region in $P$. harei also reveals long segments of synteny, another observation indicating that the transfer event was recent.

A PCR screen of the 27 TvLF genes in four additional strains of T. vaginalis yielded 105 out of the expected 108 PCR products. These 105 products were all sequenced on both strands. The inability to obtain an amplicon in the remaining three cases, despite numerous attempts, using both alternative primers designed in regions conserved between $T$. vaginalis and $P$. harei and exact match primers in sequences from PCR products in other strains, might be due to either actual gene loss, or to rearrangements resulting in loss of primer sites.
Within the TvLF region, eight additional genes are encoded in $P$. harei but were not detected in any strain of $T$. vaginalis. These genes could have been acquired either by $P$. harei after the divergence of $P$. harei and the putative $P$. harei-like donor, or after the transfer of genes to $T$. vaginalis. Alternatively, the eight additional genes could have been present in the $P$. harei-like donor and transferred to Trichomonas but subsequently have been lost in the T. vaginalis genome (Figure 2, yellow dotted, arrowed lines).

Of the 27 TvLF genes, 23 show homology and are in synteny with genes in the corresponding region of $P$. harei, and are found in all strains investigated. Three of the remaining genes (TVAG_243571, TVAG_243580 and TVAG_243650, no. 3, 5 and 19 Figures 1 and 2) are 
found in all $T$. vaginalis strains but have not been identified in the complete genome sequence of $P$. harei. They do, however, exhibit high nucleotide sequence homology to sequences found in e.g. P. rhinitidis (WP_010242155.1) and $P$. str. F0141 (WP_009345232.1), as determined from phylogenetic analyses (Additional file 2: Figure S2). These LGTs (denoted by red dotted, arrowed lines) were either: 1 ) acquired by the common ancestor of $T$. vaginalis strains in an event distinct from the uptake of TvLF, 2) acquired by the $P$. harei-like donor and transferred along with the rest of the genes of TvLF to Trichomonas; or 3) lost in P. harei subsequent to the transfer event of TvLF to T. vaginalis. TVAG_243620 and TVAG_243720, (no. 16 and 29 in Figures 1 and 2) are only found in T. vaginalis strains G3, Moz-4 and Pinna, and are products of a gene fission caused by a stop codon and a major modification, respectively.

The comparison of TvLF genes annotated in $T$. vaginalis G3 with the homologous region in $P$. harei reveals 11 genes of equal length. In 7 of the remaining 16 genes, all of which are annotated as shorter in Trichomonas than in the bacterial orthologs, alternative start codons can be chosen to achieve longer ORFs that more closely resemble the $P$. harei orthologs (Additional file 1: Table S6).

In total we have detected eight TvLF genes that have accumulated stop codons: four genes in $T$. vaginalis strains G3, Pinna and Moz-4, and four distinct genes in T. vaginalis strains Tor-A and Casu2 (Table 3 and no. 14, 16, 18, 28, 35, 40-42 in Figures 1 and 2). For example, strains Tor-A and Casu2 have two cases of full-length genes

Table 3 TvLF genes in the five investigated $T$. vaginalis strains with acquired termination codons

\begin{tabular}{llll}
\hline Affected gene & Position & Strain & Comment \\
\hline TVAG_243610 & 26605 & G3, Pinna and Moz-4 & Causes formation of TVAG_243603 and TVAG_243610 \\
TVAG_2436201,2 & 28540 & G3, Pinna and Moz-4 & Causes formation of TVAG_243620 and TVAG_243630 \\
TVAG_243640 & 30623 & Tor-A and Casu2 & \\
TVAG_243710 & 38338 & G3, Pinna and Moz-4 & Involved in the formation of TVAG_243710 and TVAG_243720 \\
TVAG_243760 & 42180 & Casu2 & \\
TVAG_243810 & 50314 & G3, Pinna and Moz-4 & \\
TVAG_243820 & 50715 & Tor-A & \\
TVAG_243830 & 51667 & Tor-A & \\
\hline
\end{tabular}

${ }^{1}$ Termination codons annotated in T. vaginalis $\mathrm{G} 3$.

${ }^{2}$ Causes formation of $T$. vaginalis G3, Pinna and Moz-4 unique genes. 
equal to the corresponding genes in $P$. harei, while the two orthologs in T. vaginalis strains G3, Pinna, and Moz-4 are divided into four ORFs due to shared stop codons disrupting the genes (No. 16 and 17 as well as No. 28 and 29 respectively, in Figure 1). Similarly, TVAG_243610, together with the new ORF TVAG_243603 (No. 14 and 15 in Figure 1), are fragments in T. vaginalis G3, Pinna and Moz-4 corresponding to the beginning and end, respectively, of $P$. harei HMPREF9286_0319. However, this gene is intact in T. vaginalis Tor-A and Casu2. Mapping the termination codons causing this differential pseudogenization onto a phylogenetic tree shows that these events occur in a parsimonious fashion (Figure 2). Common to all genes of TvLF interrupted by termination codons is that they are all involved in information processes or annotated as hypothetical proteins. None of the genes involved in metabolic processes are found to have acquired stop codons.

We have detected one major gene rearrangement (no. 28 in Figures 1 and 2) resulting in a unique gene, TVAG_243720, shared by $T$. vaginalis G3, Pinna and Moz-4. TVAG_243720 consists of the reversed end of the second half of the $P$. harei ortholog, immediately followed by a downstream deletion of approximately $400 \mathrm{bp}$. Within this $400 \mathrm{bp}$ region, which still remains intact in T. vaginalis Tor-A and Casu2, an alternative start codon is present, which results in an extension of TVAG_243730 in T. vaginalis Tor-A and Casu2, which resemble the $P$. harei ortholog with respect to both size and nucleotide sequence similarity.

In addition to the 27 annotated TvLF genes, 7 previously un-annotated TvLF ORFs were identified by sequence comparison. Five of these ORFs have high nucleotide sequence similarity to genes of $P$. harei, while two ORFs are only present in other species of Peptoniphilus. These newly discovered ORFs were given names according to the gene directly upstream the ORF. Five of the seven new ORFs are present in all species and yield high scoring similarities to other genes, providing a tentative function (Additional file 1: Table S7). The two remaining ORFs (TVAG_243601 and TVAG_243602, no. 12 and 13 in Figure 1) contain conserved domain areas suggested by the Conserved Domain Database (CDD) to be ABC-2 family transporter proteins, and are only found in the strains of $T$. vaginalis [29].

Among the 27 genes of TvLF, eight are annotated as hypothetical proteins or genes involved in uncharacterized processes. Furthermore, four are categorized as involved in metabolic processes, eight as involved in genetic information processes, and seven in environmental information processes (transport). The latter two classes are both under-represented in other surveys of LGT in protozoa $[13,30]$. The pseudogenization is most widespread among genes involved in informational processes (five out of eight) as compared to those involved in transport (one of seven) and metabolism, where all genes are still intact (Table 4 and Additional file 1: Table S5).

\section{Phylogeny}

In order to determine the potential donor of the genes of TvLF, as well as to investigate whether all genes may have one single donor, phylogenetic trees of all genes of TvLF were estimated. All trees (Additional file 2: Figure S2) support the close relationship of TvLF-sequences of T. vaginalis with bacteria of the Firmicute lineage, to the exclusion of any other bacterial or eukaryote sequence by at least one node with a minimum bootstrap support value of bs $=80 \%$. All trees confirm the hypothesis of a close relative of $P$. harei as the likely donor organism. In two gene trees (TVAG_243650 and TVAG_243580), T. vaginalis clusters with other species of the Peptoniphilus lineage; however, in neither of these cases could orthologs be detected in the genome of $P$. harei using homology searches. This indicates that the donor organism is closely related, but not identical, to P. harei, and that this currently un-sequenced firmicute likely also possesses the orthologs to TVAG_243580 and 243650. Other possible, although from a parsimony perspective considerably less likely, scenarios include multiple donor organisms of the firmicute lineage or multiple losses in $P$. harei.

To evaluate whether all genes were acquired in one single event a combined tree was produced by concatenating all sequences of TvLF and $P$. harei (with the exception of TVAG_243580 and TVAG_243650 that were coded as missing data in the analysis). The result is completely unambiguous and corroborates with high bootstrap support values the scenario shown in Figure 3.

The tree topology shows that $T$. vaginalis strains Tor-A and Casu2 form one group with high bootstrap support values (bs) from subsequent support analyses (bs $=91 \%$ ), while $T$. vaginalis strains G3, Pinna and Moz-4 form another distinct and well supported clade (bs $=100 \%$ ), in which the latter two also group together (bs $=92 \%$ ). Thorough analysis of TvLF within these five different strains of $T$. vaginalis reveals a multitude of

Table 4 Functional distribution of LGTs in TvLF compared to the average functional distribution of LGTs in protists

\begin{tabular}{lllll}
\hline Gene function & $\begin{array}{l}\text { No. genes } \\
\text { in TvLF }\end{array}$ & $\begin{array}{l}\text { No. pseudogenes } \\
\text { in TvLF }\end{array}$ & $\begin{array}{l}\text { \% in } \\
\text { TvLF }\end{array}$ & $\begin{array}{l}\text { in protists } \\
\text { i }\end{array}$ \\
\hline Metabolism & 4 & 0 & 14 & 44 \\
$\begin{array}{l}\text { Genetic information } \\
\text { processing }\end{array}$ & 8 & 5 & 28 & 6 \\
$\begin{array}{l}\text { Environmental } \\
\text { information } \\
\text { processing }\end{array}$ & 7 & 1 & 24 & 3 \\
\begin{tabular}{l} 
Hypothetical \\
\hline
\end{tabular} & 10 & 2 & & \\
\hline
\end{tabular}

a[13]. 


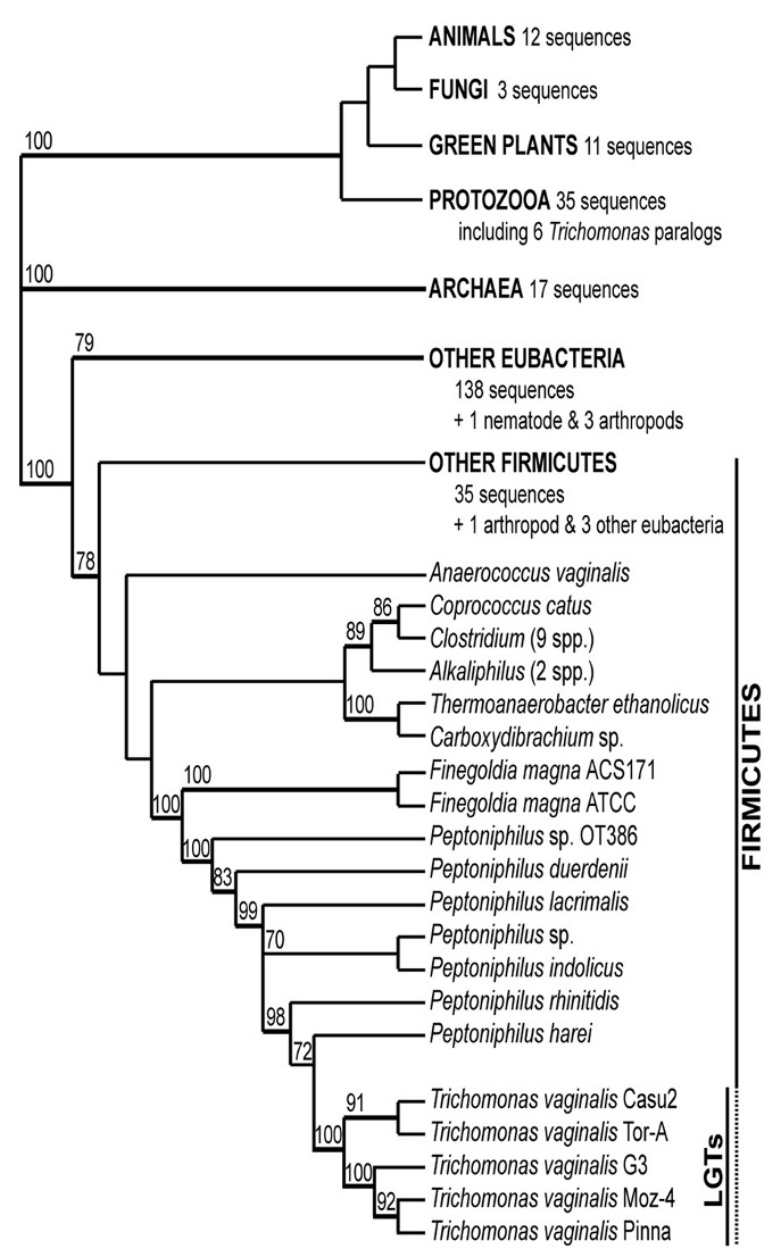

Figure 3 Summarized synthetic phylogenetic tree of TvLF. Synthetic phylogenetic tree summarizing congruent groupings retained from the analyses of separate gene support (Additional file 2: Figure S2) for the different TVLF gene homologs identified in various taxa, along with their corresponding bootstrap values. Large, well-supported clades with many representatives are reduced to placeholders, with the exception of the sections close to TVLF positions. All evaluated trees provide clear and unambiguous support for the placement of TVLF-sequences in the firmicute lineage.

rearrangements, insertion, and deletion events (Figures 1 and 2), some of which render novel initiation or termination codons. Mapping the differences in TvLF between $T$. vaginalis strains on a phylogenetic sub-tree shows that all changes are parsimonious (Figure 2). This is a further evidence for the authenticity of TvLF as an integrated part of the T. vaginalis nuclear genome.

\section{Sequence analysis}

CAI-values for the TvLF genes calculated with $T$. vaginalis codon usage data differ significantly from the values obtained for other firmicute codon usage data (Figure 4A). The average CAI for all genes of TvLF in each strain is significantly lower in $T$. vaginalis than in Firmicutes. This result is in line with the hypothesis that the TvLF was transferred recently, and that genetic information in the TvLF has not yet been molded to fit into the codon bias acting in $T$. vaginalis. The set of $T$. vaginalis housekeeping genes used as a reference has significantly higher CAI-value in $T$. vaginalis.

A cumulative GC profile was assembled to visualize the general nucleotide composition features of the TvLF. This has been suggested as a tool to indicate genomic islands or to identify LGTs [31,32]. The cumulative GC profile of scaffold DS113827 in the T. vaginalis G3 genome (Figure 4B) displays a strong segmentation (segmentation point 3, strength 90.48) at position 19,014 bp, with an increased GC content ending at position 21,591 bp. This segmentation point accords with the first Peptoniphilus-like gene, the first gene in TvLF, TVAG_243570. The strong segmentation point at 19,014 bp coinciding with the beginning of the TvLF further supports the hypothesis that this region was acquired from a foreign DNA source.

At the position of the second gene of TvLF, TVAG_ 243580, another segmentation point (point 4, strength 14.99) can be found with a small decrease in GC-content. 

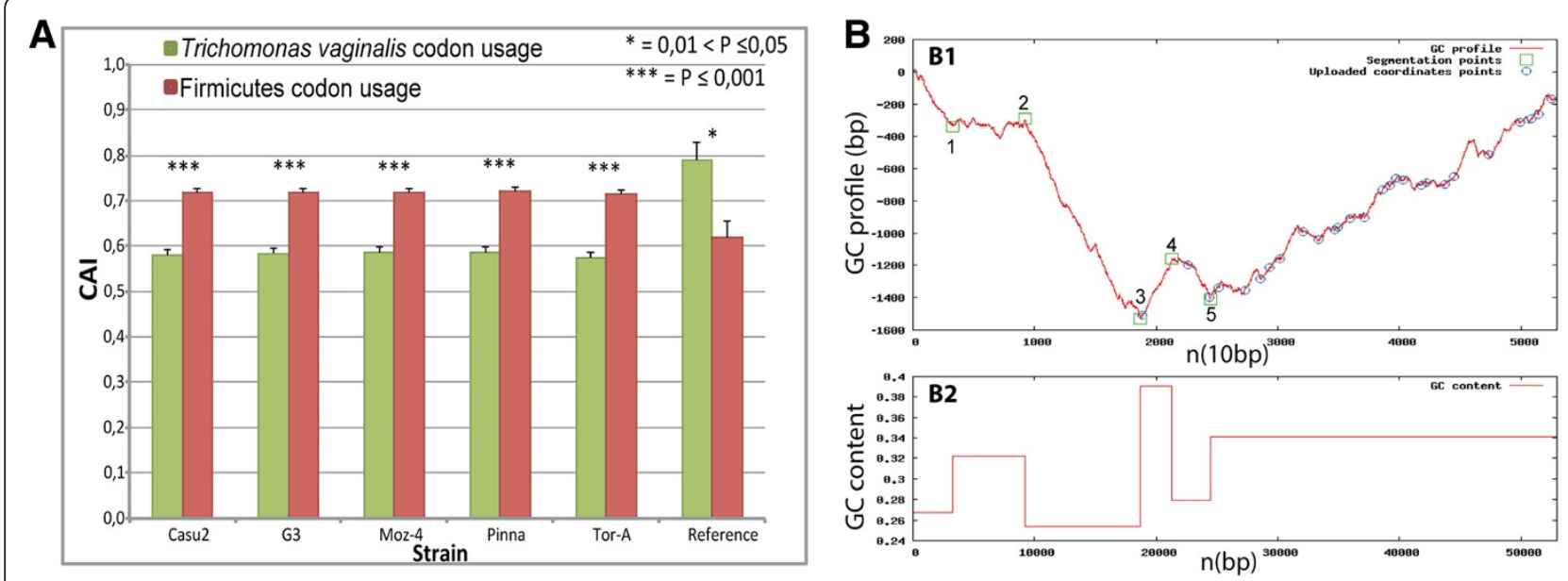

Figure 4 The average Codon Adaptation index and the cumulative GC profile for TVLF. A. The average Codon Adaptation Index (CAI) for each TVLF gene in the five T. vaginalis strains investigated. Green bars represent CAl calculated using the T. vaginalis codon usage data, and the red bars using firmicute codon usage data. Firmicute codon bias based CAl is significantly higher than $T$. vaginalis codon bias based CAI in all strains ( $p \leq 0.0001$ ). Furthermore, CAl calculated for the reference gene-set using firmicute codon bias is significantly lower than CAI calculated using $T$. vaginalis codon bias data $(0.01<P \leq 0.05)$. A pooled, two sample t-test was used to determine significance. Error bars represent standard errors of the mean. B1. Negative z' curve (cumulative GC profile) for TVLF T. vaginalis G3. Segmentation points are marked with green squares, and the positions of the bacteria-like genes are indicated with blue rings. Segmentation point 3 (segmentation strength 91.69) coincides with the beginning of the first LGT (the first P. harei-like gene, TVAG_243570). B2. The GC content distribution along the TvLF, using a 1000 bp sliding window.

This gene is not present in the genome of P. harei. However, in other species of Peptoniphilus, $P$. indolicus and $P$. rhinitidis, orthologs are retrieved with strong similarity scores at the DNA level and coherent positioning in the phylogenetic tree. These observations could indicate that the gene TVAG_243580, coding for a transposase, was acquired by LGT into the actual donor, as well as some of the other more distantly related Peptoniphilus species, after the divergence of the Peptoniphilus species but prior to the transfer-event giving rise to the TvLF. However, a more parsimonious view is that the gene was lost in $P$. harei but retained in the other species.

\section{Discussion}

Our hypothesis is that a 34,102 bp long fragment, TvLF, encompassing 27 consecutive bacteria-like genes and seven un-annotated ORFs is the result of a recent gene transfer event from one single bacterial donor, presumably a close relative to the firmicute $P$. harei. This region stands in contrast to other LGTs detected in eukaryotic parasite genomes, which are almost always single gene occurrences embedded among genes of eukaryote origin $[17,27]$.

The possibility that LGTs are initially acquired in clusters in protozoa has previously been proposed, although actual cases are rare. In Giardia intestinalis isolate GS three consecutive LGTs are found, although they are absent in Giardia intestinalis isolate WG, further advocating the hypothesis of a recent transfer [33]. In Cryptosporidium two separate pairs of genes appear to have been acquired at the same time [20]; and the ascomycete Trichoderma appears to have acquired a three-gene cluster resembling part of a nitrate assimilation pathway from a distantly related basidiomycete lineage [34]. Similar phenomena have also been observed in metazoan [35] and in plant mitochondria [36].

One reason for the apparent scattering of the typical LGTs in protozoa could be that once foreign DNA has been acquired and integrated into the host chromosome, there are two possible scenarios: loss or preservation. A newly acquired gene may degrade through mutational processes and vanish. If preserved, the LGT can relocate via internal recombination events, duplicate and evolve into a functional gene, possibly with a modified function $[37,38]$. This latter process, however, is as yet poorly understood, albeit the amelioration process has been shown to work rapidly on LGTs in bacteria, and would in these cases obliterate the compositional differences to the host genome [39]. Consequently, evaluation of compositional differences has been shown to be a poor marker for LGT [40].

The so-called "you are what you eat" hypothesis promoted by Doolittle (1998) suggests that genetic material can be incorporated into a unicellular eukaryotes genome. This may happen by chance after phagocytosis of bacteria populating the same habitat, and is supported by the fact that phagotrophs have a higher rate of LGT than non-phagotrophs $[10,11]$. Donors of LGTs in protozoa are predominantly bacteria sharing a habitat [13], making Bacteroides, Clostridium, and related species common 
contributors to $T$. vaginalis. Our findings support this theory, since $P$. harei, a firmicute and close relative of the assumed donor, shares a habitat with $T$. vaginalis in the urogenital tract and reproductive system of women [41-43]. We may further hypothesize that all of the LGTs in $T$. vaginalis that stem from, for example, the Bacteroides lineage may have been acquired in one or a few batches, in a similar mode to the TvLF, although genomic rearrangements in both donor and recipient have erased obvious evidence such as synteny.

Thus, we argue that the LGTs left in today's protozoan genomes are the successfully fixed genes, still remaining after having passed evolutionarily driven rearrangements, and evaded gene decay. If the transfer of TvLF is as recent as indicated, it becomes reasonable to assume that this process is still ongoing, and that some genes may be retained under a selective pressure, while others evolve under more relaxed constraints and are likely to be lost in the future. In the case of TvLF we identify several instances where the latter situation occurs, for example genes disrupted by internal stop codons, resulting in pseudogenes.

Furthermore, if the physical uptake of genetic material is assumed to be random, while the fixation of genes is under a selective pressure $[11,16]$, it follows that it is also reasonable that a very recent LGT-event has not yet been cleansed of obsolete material and streamlined to fit the exact requirements of the recipient organism. Such modifications have been shown to take place in bacteria during the amelioration process [39]. If we assume that similar processes are at work also in eukaryotes, then they have in TVLF presumably not yet had the time to homogenize the LGTs to resemble the remainder of the genome.

This is supported by results from the codon adaptation index (CAI) analyses for TvLF, where we show that CAI-values for genes in TvLF resemble those of the $P$. harei genome rather than those of the remainder of the T. vaginalis genome. The sequence similarity between the donor and the recipient is also higher than what is usually observed among LGTs. Similarly, calculation of cumulative $\mathrm{GC}$ values nominates the region between $T$. vaginalis specific repeated genes and TvLF, immediately adjacent to the locus of the first Peptoniphilus-like gene, as a site for incorporation of foreign DNA. Such strong segmentation points are described, for instance, in genomic islands found in bacteria with uniform GC-content $[44,45]$.

The functional categorization of previously detected LGTs in bacteria and eukaryotes shows that most LGTs are active in metabolic processes, while informational genes are rare $[11,13,16]$. This phenomenon may reflect that, although the uptake happens by chance, the fixation does not, and that LGT predominantly is important for adaptation processes such as utilization of new metabolites, but less important for optimization of informational processes already encoded for by the cell.

In TvLF we have identified eight genes known to be active in genetic informational processes, such as transcription and cell cycle control; however, the majority of these have accumulated stop codons in one or more of the strains (five out of eight). This observation strengthens both the assumption that the TvLF region is evolving rapidly to remove undesirable genes and that the informational genes, to a certain extent, are within this less desirable category of genes. An additional observation that further confirms this hypothesis is that TvLF harbors only four genes known to be involved in metabolism, and all of these genes are intact. All four genes have previously been shown to be expressed [46], thus indicating that they may be functionally active.

More surprisingly, the TvLF encompass seven genes involved in transport, whereof six are intact in all of the strains investigated. A closer look at the functional annotation of these transport genes reveals that several are homologous to genes involved in antibiotic resistance in bacteria. Development of antibiotic resistance in bacteria is often achieved via LGT, but so far, to our knowledge, no such cases have been reported in protozoa. Whether some or all of these genes are actually active in $T$. vaginalis remains to be investigated.

Furthermore, in TvLF we have also observed two transposases, genes that are associated with transposition of genetic material, and may thus, hypothetically, be involved in the incorporation process during the actual uptake of TvLF. These transposases are not present in the corresponding region in $P$. harei, but are found in other Firmicutes of the Peptoniphilus lineage.

\section{Conclusions}

In this study, the comprehensive comparative sequence analysis of the TvLF in five different strains of Trichomonas and the putative bacterial donor, Peptoniphilus, reveals an unusually high degree of nucleotide sequence similarity and synteny, supporting the hypothesis that TvLF is the result of a single, recent transfer event. Repeated attempts to amplify genes from the TvLF in other Trichomonas species, such as $T$. gallinae and $T$. tenax, have proven unsuccessful, indicating that the transfer occurred after the divergence of $T$. vaginalis from other trichomonads. Furthermore, an array of rearrangements, insertion, and deletion events - some of which render novel initiation or termination codons - are also found, indicating that part of TvLF is in a state of rapid evolutionary change. Mapping these features of TvLF onto a phylogenetic tree based on concatenated sequences of all genes in TvLF demonstrates that all of the features identified in this study can be explained in a parsimony framework. 
Among the LGTs of TvLF, several are functionally annotated as genetic information processing genes, a functional category of genes that are under-represented among LGTs detected previously. However, most of these informational genes have been disrupted by internal stop codons. Uptake of long clusters of genes contributes a broad selection of novel functions to pick and choose from. The strategy to, at the same time, preserve the most beneficial genes only, may be the advantageous strategy to effectively gain new abilities.

Altogether, this study of the unique TvLF region demonstrates how the fixation process of recently acquired genetic material is shaped to resemble the norm for the microbial eukaryote transferome.

\section{Methods}

\section{Organisms and cell culture}

Four strains of $T$. vaginalis ( $T$. vaginalis Casu2 SS22, T. vaginalis Moz-4 MPM4, T. vaginalis Tor-A TO 01, T. vaginalis Pinna SS28) were kindly provided by Dr. Pier Luigi Fiori at the Dept. of Biomedical Sciences, Division of Experimental and Clinical Microbiology, University of Sassari, Sassari, Italy; and T. vaginalis G3 DNA was provided from Prof. Robert Hirt at the Institute for Cell and Molecular Bioscience, Newcastle University. In addition, $T$. vaginalis $\mathrm{T} 1$ and $\mathrm{P} 9, \mathrm{~T}$. gallinae GCB P41 and T. tenax HS4 were provided by J. Tachezy, Department of Parasitology, Charles University, Prague, Czech Republic (Table 1). Cultures of trichomonads were grown as described previously [47]. DNA from all strains was extracted with DNeasy Blood and Tissue kit (Qiagen, Valencia, CA) and high Pure PCR Template Preparation Kit (Roche, Basel, $\mathrm{CH}$ ) in accordance with the manufacturer's instructions.

\section{PCR screen}

PCR amplifications were performed in $50 \mu$ l reaction volume using either Mastercycler Personal (Eppendorf, Hamburg, D) or $\mathrm{C}_{1000}{ }^{\mathrm{m}}$ Thermal Cycler (Bio-Rad, Hercules, CA): $75 \mathrm{mM}$ Tris- $\mathrm{HCl}$ pH 8.5, $20 \mathrm{mM}\left(\mathrm{NH}_{4}\right)$ ${ }_{2} \mathrm{SO}_{4}, 2 \mathrm{mM} \mathrm{MgCl}_{2}, 0.1 \%$ Tween $20^{\circ}, 0.2 \mathrm{mM}$ dNTPs and 1.25 U Ampliqon Taq polymerase (VWR International, Radnor, PN), 0,2-0,4 $\mu \mathrm{M}$ of each primer (Sigma-Aldrich, St. Louis, MO), 10-100 ng template and double distilled, sterile filtered using Milli-Q filter (Merck Millipor, Billerica, MA) water up to $50 \mu \mathrm{l}$. PCR amplification always began with one hold cycle at $95^{\circ} \mathrm{C}$ for 3 minutes followed by $30-35$ cycles of $95^{\circ} \mathrm{C}$ for $30 \mathrm{~s}$; primer melting point $(\mathrm{Tm})$ minus $3-5^{\circ} \mathrm{C}$ for approximately $1 \mathrm{~min} /$ $1000 \mathrm{bp}$ product; and $72^{\circ} \mathrm{C}$ for $1 \mathrm{~min}$. The amplification always ended with $72^{\circ} \mathrm{C}$ for $10 \mathrm{~min}$ as a final elongation step, followed by $4^{\circ} \mathrm{C}$ until manually shut down. Primers were designed in Geneious v.5 and v.6 (Biomatters,
Auckland, NZ) using the Primer3 algorithm [48,49]. Primer specification can be found in Additional file 1: Table S8.

PCR products were analyzed on-chip with a MultiNA (Shimadzu, Kyoto, J); and positive amplifications were purified with GenElute ${ }^{\mathrm{TM}}$ PCR Clean-Up kit (Sigma-Aldrich), sequenced through Standard-Seq by Macrogen (Macrogen Corporation, Amsterdam, NL) and assembled using Geneious. All assemblies were based on sequences from both strands with a minimum of two-fold coverage. Measures to avoid errors consisted of by eye examination of assemblies, before consensus sequences were deposited in GenBank.

\section{Sequence analysis}

A comprehensive search for potential un-annotated ORFs (open reading frames) and other sequence ambiguities in the entire scaffold DS113827 was performed and compared to the corresponding region of $P$. harei, using Geneious and National Center for Biotechnology Information (NCBI) Basic Local Alignment Search Tool, BLAST [50].

A cumulative $\mathrm{GC}$ profile was assembled to visualize the general composition features of the TvLF using GC-content data [31]. Input parameters were set to the default, with the exception of the 'halting parameter', which was adjusted to 10 . In the GC plot a coordinate file was incorporated consisting of T. vaginalis G3 LGTs positions, to visualize the correlation between the GC-shifts and the spatial distribution of the bacteria-like genes.

The codon adaptation index (CAI) is suggested as a way to measure synonymous codon usage bias $[51,52]$. $T$. vaginalis has been suggested to have a biased codon usage, and therefore becomes suitable for CAI calculation [53]. CAI estimates have also been used to evaluate whether the genetic material is recently acquired, as the host should not have had time to adapt recently retrieved material to fit its own codon usage [54]. In this particular case, however, the time for acquisition will be identical for all genes in TvLF, and thus this aspect becomes less important.

CAI were calculated for each TvLF gene for all T. vaginalis strains using the web based CAI-Calculator by Puigbo and co-workers [55]. Also, a set of four T. vaginalis housekeeping genes was used as reference: TVAG_343390: mannose-6-phosphate isomerase; TVAG_299450: alanyltRNA synthetase; TVAG_258340: family T2 asparaginaselike threonine peptidase; and TVAG_054490: tryptophanase [56]. As codon usage data, the codon usage table for T. vaginalis based on 189 CDSs $(65,401$ codons) was employed. Because the absence of a $P$. harei codon usage table, two other closely related (Figure 3) firmicute bacteria codon usage tables, Clostridium thermocellum ATCC 27405 based on 3191 CDS's (1,072,649 codons) and Lactobacillus gasseri ATCC 33323 based on 1755 CDSs (558,761 codons) served as template to calculate CAI and 
the average CAI-values from the two different bacteria were used. The codon usage tables were acquired from public online resources (www.kazusa.or.jp/codon/).

In order to evaluate the evolutionary adaptation, a comparison was made between the average CAI-values on the TvLF genes calculated with $T$. vaginalis codon usage and the average CAI-values obtained from calculations made with both Clostridium thermocellum and Lactobacillus gasseri.

\section{Phylogenetic analyses}

Phylogenetic analyses of the different genes in TvLF were executed in order to determine their evolutionary origin. TvLF genes were used as the query sequence to perform homology searches using the blastx-algorithm [50] on the non-redundant protein sequences (NR) database (http://blast.ncbi.nlm.nih.gov/Blast), and the relevant homologs were collected. The matrices aimed to include homologs from five firmicute bacteria, 20 bacteria from other phyla, and 20 eukaryotic organisms of different phyla.

Alignments were performed using ClustalW algorithm [57] and subsequently inspected and edited manually using Geneious. The phylogenetic analyses of the obtained matrices were performed using PAUP* v. 4.0b10 software (Sinauer Assoc. Inc., Sunderland, MA; [58] running under Mac OS X on an MacBook Pro with 8 GB of memory available, SSD and $3.8 \mathrm{GHz}$ Intel Core i7 processor (Apple, Cupertino, CA). The analyses were executed under the maximum parsimony criterion [59], and included 1,000 random addition sequence replicates followed by TBR branch-swapping, followed by the calculation of a strict consensus tree. Tree support was estimated using a bootstrap approach [60] with 100 bootstrap replicates, each followed by 100 random addition sequence replicates, followed by SPR branch-swapping. All strict consensus trees and general analysis statistics are supplied in Additional file 2: Figure S2.

\section{Data access}

Generated data are uploaded to GenBank and were allocated the following accession numbers; KF269355-KF269530. http://www.ncbi.nlm.nih.gov/nuccore/KF269355.1 - http:// www.ncbi.nlm.nih.gov/nuccore/KF269530.1.

The data set supporting the results of this article is available in the Dryad repository; http://datadryad.org/ resource/doi:10.5061/dryad.30r6p [61].

\section{Additional files}

Additional file 1: Table S1. Listing the Trichomonas unique genes paralogs located on the DS113827 contig, directly adjacent to the TVLF. Table S2. Contains information regarding the annotated gaps in contig DS113827. Table S3. Lists the 16 s rDNA primers used for detection of posible contaminant bactera. Table S4. Contains detailed information on extended ORFS in TVLF; and Table S5. Has a detailed list of the genes of TVLF. Table S6. Containes a list with abbreviations found in the gene map (Figure 1). Table S7. Contains data on newly discovered ORFs in TVLF. Table S8. Lists the oligonucleotides used in this study.

Additional file 2: Figure S2. Summary and results from phylogenetic analyses of all 27 genes of the TVLF, and analysis to elucidate relationship between Trichomonas vaginalis strains.

\section{Competing interests}

The authors declare that they have no competing interests.

\section{Authors' contributions}

Ås performed the experimental work and the bulk of the bioinformatic analyses, complemented by analyses from CA and AB. CA conceived and coordinated the project. All authors wrote and edited the manuscript. All authors read and approved the final manuscript.

\section{Acknowledgments}

This work was supported by a FORMAS grant (grant number 2008-1366) to Cecilia Alsmark, including support for Åke Strese. The funders had no role in study design, data collection and analysis, decision to publish, or preparation of the manuscript. We are indebted to Dr. Luigi Fiori (Dept. of Biomedical Sciences, Division of Experimental and Clinical Microbiology, University of Sassari, Sassari, Italy), Dr. Robert Hirt (Institute for Cell and Molecular Biosciences, Newcastle University, Newcastle, United Kingdom), and Prof. Jan Tachezy and Dr. Zuzana Zubáčová (Department of Parasitology, Charles University, Prague, Czech Republic) for their kind donations of Trichomonas DNA.

Received: 17 January 2014 Accepted: 27 May 2014

Published: 5 June 2014

\section{References}

1. World Health Organization DoRHaR: Chlamydia trachomatis, Neisseria gonorrhoeae, syphilis and Trichomonas vaginalis. Methods and Results Used by WHO to Generate 2005 Estimates. In Prevalence and Incidence of Selected Sexually Transmitted Infections. Geneva: WHO Press; 2011.

2. Petrin D, Delgaty $K$, Bhatt R, Garber G: Clinical and microbiological aspects of Trichomonas vaginalis. Clin Microbiol Rev 1998, 11:300-317.

3. McClelland RS, Sangare L, Hassan WM, Lavreys L, Mandaliya K, Kiarie J, Ndinya-Achola J, Jaoko W, Baeten JM: Infection with Trichomonas vaginalis increases the risk of HIV-1 acquisition. J Infect Dis 2007, 195:698-702.

4. Ryan CM, de Miguel N, Johnson PJ: Trichomonas vaginalis: current understanding of host-parasite interactions. Essays Biochem 2011, 51:161-175.

5. Lumsden $\mathrm{WH}$, Robertson $\mathrm{DH}$, Heyworth $\mathrm{R}$, Harrison $\mathrm{C}$ : Treatment failure in Trichomonas vaginalis vaginitis. Genitourin Med 1988, 64:217-218.

6. Zubacova Z, Cimburek Z, Tachezy J: Comparative analysis of trichomonad genome sizes and karyotypes. Mol Biochem Parasitol 2008, 161:49-54.

7. de Koning AP, Brinkman FS, Jones SJ, Keeling PJ: Lateral gene transfer and metabolic adaptation in the human parasite Trichomonas vaginalis. Mol Biol Evol 2000, 17:1769-1773.

8. McGowan C, Fulthorpe R, Wright A, Tiedje JM: Evidence for interspecies gene transfer in the evolution of 2,4-dichlorophenoxyacetic acid degraders. Appl Environ Microbiol 1998, 64:4089-4092.

9. Radstrom P, Fermer C, Kristiansen BE, Jenkins A, Skold O, Swedberg G: Transformational exchanges in the dihydropteroate synthase gene of Neisseria meningitidis: a novel mechanism for acquisition of sulfonamide resistance. J Bacteriol 1992, 174:6386-6393.

10. Keeling PJ, Palmer JD: Horizontal gene transfer in eukaryotic evolution. Nat Rev Genet 2008, 9:605-618.

11. Doolittle WF: You are what you eat: a gene transfer ratchet could account for bacterial genes in eukaryotic nuclear genomes. Trends Genet 1998, 14:307-311.

12. Clarke M, Lohan AJ, Liu B, Lagkouvardos I, Roy S, Zafar N, Bertelli C, Schilde C, Kianianmomeni A, Bürglin TR, Frech C, Turcotte B, Kopec KO, Synnott JM, Choo C, Paponov I, Finkler A, Tan CSH, Hutchins AP, Weinmeier T, Rattei T, Chu JSC, Gimenz G, Irima M, Rigden DJ, Fitzpatrick DA, Lobrenzo-Morales J, Bateman A, Chiu CH, Tang P, et al: Genome of Acanthamoeba castellanii 
highlights extensive lateral gene transfer and early evolution of tyrosine kinase signaling. Genome Biol 2013, 14:R11.

13. Alsmark C, Foster PG, Sicheritz-Ponten T, Nakjang S, Embley TM, Hirt RP: Patterns of prokaryotic lateral gene transfers affecting parasitic microbial eukaryotes. Genome Biol 2013, 14:R19.

14. Carlton JM, Hirt RP, Silva JC, Delcher AL, Schatz M, Zhao Q, Wortman JR, Bidwell SL, Alsmark UC, Besteiro S, Sicheritz-Ponten T, Noel CJ, Dacks JB, Foster PG, Simillion C, Van de Peer Y, Miranda-Saavedra D, Barton GJ, Westrop GD, Müller S, Dessi D, Fiori PL, Ren Q, Paulsen I, Zhang H, Bastida-Corcuera FD, Simoes-Barbosa A, Brown MT, Hayes RD, Mukherjee M, et al: Draft genome sequence of the sexually transmitted pathogen Trichomonas vaginalis. Science 2007, 315:207-212.

15. Alsmark UC, Sicheritz-Ponten T, Foster PG, Hirt RP, Embley TM: Horizontal gene transfer in eukaryotic parasites: a case study of Entamoeba histolytica and Trichomonas vaginalis. Meth Mol Biol 2009, 532:489-500.

16. Jain R, Rivera MC, Lake JA: Horizontal gene transfer among genomes: the complexity hypothesis. Proc Natl Acad Sci USA 1999, 96:3801-3806.

17. Loftus B, Anderson I, Davies R, Alsmark UC, Samuelson J, Amedeo P, Roncaglia P, Berriman M, Hirt RP, Mann BJ, Nozaki T, Suh B, Pop M, Duchene M, Ackers J, Tannich E, Leippe M, Hofer M, Bruchhaus I, Willhoeft U, Bhattacharya A, Chillingworth T, Churcher C, Hance Z, Harris B, Harris D, Jagels $K$, Moule $S$, Mungall $K$, Ormond $D$, et al: The genome of the protist parasite Entamoeba histolytica. Nature 2005, 433:865-868.

18. Yusof A, Kumar S: Ultrastructural changes during asexual multiple reproduction in Trichomonas vaginalis. Parasitol Res 2012, 110:1823-1828.

19. Tibayrenc M, Kjellberg F, Ayala FJ: A clonal theory of parasitic protozoa: the population structures of Entamoeba, Giardia, Leishmania, Naegleria, Plasmodium, Trichomonas, and Trypanosoma and their medical and taxonomical consequences. Proc Natl Acad Sci U S A 1990, 87:2414-2418.

20. Huang J, Mullapudi N, Lancto CA, Scott M, Abrahamsen MS, Kissinger JC: Phylogenomic evidence supports past endosymbiosis, intracellular and horizontal gene transfer in Cryptosporidium parvum. Genome Biol 2004 5:R88.

21. Li ZW, Shen YH, Xiang ZH, Zhang Z: Pathogen-origin horizontally transferred genes contribute to the evolution of Lepidopteran insects. BMC Evol Biol 2011, 11:356.

22. Vancini RG, Benchimol M: Entry and intracellular location of Mycoplasma hominis in Trichomonas vaginalis. Arch Microbiol 2008, 189:7-18.

23. Dessi D, Delogu G, Emonte E, Catania MR, Fiori PL, Rappelli P: Long-term survival and intracellular replication of Mycoplasma hominis in Trichomonas vaginalis cells: potential role of the protozoon in transmitting bacterial infection. Infect Immun 2005, 73:1180-1186.

24. Germain M, Krohn MA, Hillier SL, Eschenbach DA: Genital flora in pregnancy and its association with intrauterine growth retardation. J Clin Microbiol 1994, 32:2162-2168.

25. Aurrecoechea C, Brestelli J, Brunk BP, Carlton JM, Dommer J, Fischer S, Gajria B, Gao X, Gingle A, Grant G, Harb OS, Heiges M, Innamorato F, lodice J, Kissinger JC, Kraemer A, Li W, Miller JA, Morrison HG, Nayak V, Pennington C, Pinney DF, Roos DS, Ross C, Stoeckert CJ, Sullivan S, Treatman C, Wang H: GiardiaDB and TrichDB: integrated genomic resources for the eukaryotic protist pathogens Giardia lamblia and Trichomonas vaginalis. Nucleic Acids Res 2009, 37:D526-D530.

26. Malik SB, Brochu CD, Bilic I, Yuan J, Hess M, Logsdon JM Jr, Carlton JM: Phylogeny of parasitic parabasalia and free-living relatives inferred from conventional markers vs. Rpb1, a single-copy gene. PloS One 2011, 6:e20774.

27. Morrison HG, McArthur AG, Gillin FD, Aley SB, Adam RD, Olsen GJ, Best AA, Cande WZ, Chen F, Cipriano MJ, Davids BJ, Dawson SC, Elmendorf HG, Hehl AB, Holder ME, Huse SM, Kim UU, Lasek-Nesselquist E, Manning G, Nigam A, Nixon JE, Palm D, Passamaneck NE, Prabhu A, Reich Cl, Reiner DS, Samuelson J, Svard SG, Sogin ML: Genomic minimalism in the early diverging intestinal parasite Giardia lamblia. Science 2007, 317:1921-1926.

28. Berriman M, Ghedin E, Hertz-Fowler C, Blandin G, Renauld H, Bartholomeu DC, Lennard NJ, Caler E, Hamlin NE, Haas B, Böhme U, Hannick L, Aslett MA, Shallom J, Marcello L, Hou L, Wickstead B, Alsmark UC, Arrowsmith C, Atkin RJ, Barron AJ, Bringaud F, Brooks K, Carrington M, Cherevach I, Chillingworth TJ, Churcher C, Clark LN, Corton CH, Cronin A: The genome of the African trypanosome Trypanosoma brucei. Science 2005, 309:416-422.

29. Marchler-Bauer A, Lu S, Anderson JB, Chitsaz F, Derbyshire MK, DeWeeseScott C, Fong JH, Geer LY, Geer RC, Gonzales NR, Gwadz M, Hurwitz DI, Jackson JD, Ke Z, Lanczycki CJ, Lu F, Marchler GH, Mullokandov M,
Omelchenko MV, Robertson CL, Song JS, Thanki N, Yamashita RA, Zhang D, Zhang N, Zheng C, Bryant SH: CDD: a conserved domain database for the functional annotation of proteins. Nucleic Acids Res 2011, 39:D225-D229.

30. Andersson JO, Sjogren AM, Davis LA, Embley TM, Roger AJ: Phylogenetic analyses of diplomonad genes reveal frequent lateral gene transfers affecting eukaryotes. Curr Biol 2003, 13:94-104.

31. Gao F, Zhang CT: GC-Profile: a web-based tool for visualizing and analyzing the variation of GC content in genomic sequences. Nucleic Acids Res 2006, 34:W686-W691

32. Hentschel U, Hacker J: Pathogenicity islands: the tip of the iceberg. Microb Infect 2001, 3:545-548.

33. Franzen O, Jerlstrom-Hultqvist J, Castro E, Sherwood E, Ankarklev J, Reiner DS, Palm D, Andersson JO, Andersson B, Svard SG: Draft genome sequencing of Giardia intestinalis assemblage B isolate GS: is human giardiasis caused by two different species? PLoS Pathog 2009, 5:e1000560.

34. Slot JC, Hibbett DS: Horizontal transfer of a nitrate assimilation gene cluster and ecological transitions in fungi: a phylogenetic study. PLoS One 2007, 2:e1097.

35. Gladyshev EA, Meselson M, Arkhipova IR: Massive horizontal gene transfer in bdelloid rotifers. Science 2008, 320:1210-1213.

36. Rice DW, Alverson AJ, Richardson AO, Young GJ, Sanchez-Puerta MV, Munzinger J, Barry K, Boore JL, Zhang Y, dePamphilis CW, Knox EB, Palmer JD: Horizontal transfer of entire genomes via mitochondrial fusion in the angiosperm Amborella. Science 2013, 342:1468-1473.

37. Hooper SD, Berg OG: Duplication is more common among laterally transferred genes than among indigenous genes. Genome Biol 2003, 4:R48.

38. Frank AC, Alsmark CM, Thollesson M, Andersson SG: Functional divergence and horizontal transfer of type IV secretion systems. Mol Biol Evol 2005, 22:1325-1336.

39. Lawrence JG, Ochman H: Amelioration of bacterial genomes: rates of change and exchange. J Mol Evol 1997, 44:383-397.

40. Ragan MA: On surrogate methods for detecting lateral gene transfer. FEMS Microbiol Lett 2001, 201:187-191.

41. Nikolaitchouk N, Andersch B, Falsen E, Strombeck L, Mattsby-Baltzer I: The lower genital tract microbiota in relation to cytokine-, SLPI- and endotoxin levels: application of checkerboard DNA-DNA hybridization (CDH). APMIS 2008, 116:263-277.

42. Wang X, Buhimschi CS, Temoin S, Bhandari V, Han YW, Buhimschi IA Comparative microbial analysis of paired amniotic fluid and cord blood from pregnancies complicated by preterm birth and early-onset neonatal sepsis. PloS One 2013, 8:e56131.

43. Schwebke JR, Burgess D: Trichomoniasis. Clin Microbiol Rev 2004, 17:794-803. table of contents.

44. Zhang CT, Zhang R: Genomic islands in Rhodopseudomonas palustris. Nat Biotechnol 2004, 22:1078-1079.

45. Zhang $R$, Zhang $C T$ : A systematic method to identify genomic islands and its applications in analyzing the genomes of Corynebacterium glutamicum and Vibrio vulnificus CMCP6 chromosome I. Bioinformatics 2004, 20:612-622.

46. Huang KY, Chen YY, Fang YK, Cheng WH, Cheng CC, Chen YC, Wu TE, Ku FM, Chen SC, Lin R, Tang P: Adaptive responses to glucose restriction enhance cell survival, antioxidant capability, and autophagy of the protozoan parasite Trichomonas vaginalis. Biochim Biophys Acta 1840, 2014:53-64

47. Diamond LS: The establishment of various trichomonads of animals and man in axenic cultures. J Parasitol 1957, 43:488-490.

48. Untergasser A, Cutcutache I, Koressaar T, Ye J, Faircloth BC, Remm M, Rozen SG: Primer3-new capabilities and interfaces. Nucleic Acids Res 2012, 40:e115.

49. Koressaar T, Remm M: Enhancements and modifications of primer design program Primer3. Bioinformatics 2007, 23:1289-1291.

50. Altschul SF, Gish W, Miller W, Myers EW, Lipman DJ: Basic local alignment search tool. J Mol Biol 1990, 215:403-410.

51. Sharp PM, Li WH: The Codon adaptation index - a measure of directional synonymous codon usage bias, and its potential applications. Nucleic Acids Res 1987, 15:1281-1295.

52. Gouy M, Gautier C: Codon usage in bacteria: correlation with gene expressivity. Nucleic Acids Res 1982, 10:7055-7074.

53. Mclnerney JO: Codon usage patterns in Trichomonas vaginalis. Eur $J$ Protistol 1997, 33:266-273. 
54. Castillo-Ramirez S, Vazquez-Castellanos JF, Gonzalez V, Cevallos MA: Horizontal gene transfer and diverse functional constrains within a common replication-partitioning system in Alphaproteobacteria: the repABC operon. BMC Genom 2009, 10:536.

55. Puigbo P, Bravo IG, Garcia-Vallve S: CAlcal: a combined set of tools to assess codon usage adaptation. Biol Direct 2008, 3:38.

56. Cornelius DC, Robinson DA, Muzny CA, Mena LA, Aanensen DM, Lushbaugh WB, Meade JC: Genetic characterization of Trichomonas vaginalis isolates by use of multilocus sequence typing. I Clin Microbiol 2012, 50:3293-3300.

57. Thompson JD, Higgins DG, Gibson TJ: CLUSTAL W: improving the sensitivity of progressive multiple sequence alignment through sequence weighting, position-specific gap penalties and weight matrix choice. Nucleic Acids Res 1994, 22:4673-4680

58. Swofford DL: PAUP*. Phylogenetic Analysis using Parsimony (*and other Methods), 4th edition. Sunderland: Sinauer Associates; 2003.

59. Fitch WM: Toward defining the course of evolution: minimum change for a specific tree topology. Syst Zool 1971, 20:406-416.

60. Felsenstein J: Confidence-limits on phylogenies - an approach using the bootstrap. Evolution 1985, 39:783-791.

61. Strese $\AA$, Backlund A, Alsmark C: A recently transferred cluster of bacterial genes in Trichomonas vaginalis - lateral gene transfer and the fate of acquired genes. BMC Evol Biol 2014, http://datadryad.org/doi:10.5061/ dryad.30r6p.

doi:10.1186/1471-2148-14-119

Cite this article as: Strese et al:: A recently transferred cluster of bacterial genes in Trichomonas vaginalis - lateral gene transfer and the fate of acquired genes. BMC Evolutionary Biology 2014 14:119.

\section{Submit your next manuscript to BioMed Central and take full advantage of:}

- Convenient online submission

- Thorough peer review

- No space constraints or color figure charges

- Immediate publication on acceptance

- Inclusion in PubMed, CAS, Scopus and Google Scholar

- Research which is freely available for redistribution 